\title{
WHAT IS THE MEANING OF FUZZY COSTS IN FUZZY TRAFFIC ASSIGNMENT MODELS?
}

\author{
Vincent Henn \\ LICIT (INRETS-ENTPE), rue Maurice Audin \\ 69518 Vaulx-en-Velin cedex, France \\ Vincent.Henn@entpe.fr
}

\section{INTRODUCTION}

For several years, much attention has been paid to emerging advanced traffic information systems (ATIS). Such systems, giving information on the traveling conditions to drivers, are supposed to enhanced the level of service of roads and reduce congestion by making some people divert from congested areas. Lot of papers have dealt with modeling the impact of such systems on the individual route choice process and on the traffic assignment on a network. Several of them have the common idea to use fuzzy logic ${ }^{1}$, arguing that route choice does involve imprecision and uncertainty, which are especially modified by information. In this paper, we are interested in such fuzzy models and, more precisely, we will explore the type of fuzziness they use.

Fuzzy traffic assignment models can be roughly separated into two types. Models of the first class mostly derive from the initial work of Lotan [11,12] and are based on fuzzy rules and on the classical tools of fuzzy control. They handle rules such as: "if travel time on route 1 is very short and travel time on route 2 is intermediate, then I will certainly choose route 1 ". Those rules are fired simultaneously and conclusions are gathered to extract the driver decision. Such models take advantage of the well-known ease of building and robustness of fuzzy controllers. A second type of model is based on the evaluation of the routes with fuzzy costs and the comparison of those costs in the setting of possibility theory or with comparison tools that can be demonstrated to be equivalent (see for example [9]).

So, in both approaches, the principle is first to evaluate the costs of the routes and then choose the best one. They both benefit from the fuzzy logic advantages and have shown the interest to use them, but questions still remain unsolved: Where do the fuzzy costs come from? How are they constructed?

Indeed, fuzzy costs are generally ill-defined and authors only write some things like "we use a triangular fuzzy number", without more precise definition, arguing some "expertise" to construct the membership functions, etc.

It is our opinion that such answers are not acceptable and several issues can -and have to- be considered regarding the membership functions. We think that the problem of the construction of those functions cannot be answered directly but we have first to fully understand what is the meaning of fuzzy costs in such models, so that we can construct them later.

We propose in this paper to explore the semantic of fuzzy costs and search what is hidden behind them. We will show that, once their meaning has been made clear, the

\footnotetext{
${ }^{1}$ By "fuzzy logic", we mean all the work done after Zadeh initial paper [18], encompassing fuzzy sets, fuzzy rules (the only "logic" part of fuzzy logic, strictly speaking) as well as possibility theory, etc.
} 
building of their membership functions is almost straightforward. As a consequence, we will also show that fuzzy costs cannot be handled in any way, precisely because of their semantic.

Finally, we will propose a general framework for a comprehensive handling of the different aspects of fuzziness that can appear in the route choice process.

\section{WHAT IS HIDDEN BEHIND FUZZY COSTS?}

Actually, fuzzy costs can cover different type of reality and may have different meanings (see [7] for a synthesis on the semantics of fuzzy sets).

\subsection{Similarity}

The first semantic given to fuzzy costs is linked with similarity. Two costs are difficult to distinguish from a driver point of view when they are too close. Actually, driver cannot distinguish very precisely different costs and (s)he does not care of this distinction. So, we can suppose that drivers handle imprecise fuzzy costs rather than crisp ones. It is important to understood that this imprecision is due to the non-capacity or non-willingness of drivers to distinguish between different values for the same cost. Then, the membership function of the cost $\widetilde{C}_{i}$ of route $i$ is linked to the degree of similarity between $x$ and a typical element of $\widetilde{C}_{i}$ which is the real cost $C_{i}$. It can be, for example, constructed with a similarity (or proximity) measure $S$, a function from $\mathfrak{R} \times \mathfrak{R}$ into $[0 ; 1]$ verifying [19]:

$$
\begin{aligned}
\forall x \in \Re, \quad S(x, x)=1 \\
\forall x, y \in \Re, \quad S(x, y)=S(y, x) \\
\forall x, y, z \in \Re, \quad S(x, z) \geq \min \{S(x, y) ; S(y, z)\}
\end{aligned}
$$

The membership function of the fuzzy costs is then defined by:

$$
\forall x \in \Re, \mu_{\widetilde{C}_{i}}=S\left(x, C_{i}\right)
$$

An example of a similarity function that can be used is the following function with parameter $\epsilon$ :

$$
S(x, y)= \begin{cases}1-\frac{|x-y|}{\epsilon} & \text { if }|x-y| \leq \epsilon \\ 0 & \text { otherwise }\end{cases}
$$

\subsection{Preference}

Another sort of fuzziness that can be encountered in traffic assignment is linked to preference. Indeed, almost every model assumes that drivers are rationale and try to minimize their travel cost. But rationality of drivers is not so strict and optimality of the route choice is rather fuzzy. Thus preference can be modeled by a fuzzy set representing the objective which driver is trying to achieve by choosing his/her route. Let us introduce a preference measure $\phi$ over the set of available routes. $\phi\left(C_{i}\right)$ is defined to give the grade of preference for the route $i$ with cost $C_{i}$ given that the costs on other routes are $C_{1} \ldots C_{n}$. Such a preference measure can be constructed on a binary 
preference relation between two costs [14]. It verifies that $\max _{i} \phi\left(C_{i}\right)=1$ and $\phi\left(C_{i}\right)>$ $\phi\left(C_{j}\right)$ if $C_{i}<C_{j}$. A fuzzy set $\widetilde{C}^{*}$ can be constructed based on such a measure, with the following membership function:

$$
\forall x \in \Re, \mu \widetilde{C}^{*}(x)=\phi(x)
$$

This fuzzy set is to be seen as the objective which drivers are trying to reach. Note that the type of fuzziness encountered here is different from in the previous section; here it is only a question of preference.

\subsection{Uncertainty}

Finally, a third type of fuzziness can be used, based on uncertainty in the prediction of travel costs, due to unpredictable events (incidents for example) or due to the difficulty of predicting precisely the time to reach one's destination (because of dynamical aspects of traffic for example). Hence, costs can be modeled with fuzzy sets $\widetilde{C}_{i}$ of a third type where $\mu \widetilde{C}_{i}(x)$ represents the possibility that a given cost $x$ might be encountered on route $i$ :

$$
\forall x \in \Re, \mu \widetilde{C}_{i}=\pi(x)=\operatorname{Poss}\left(C_{i}=x\right)
$$

\subsection{Generalized fuzzy cost in recurrent situations}

As a matter of fact, those different semantics (similarity, preference and uncertainty) are usually considered all together. The fuzzy costs encountered in the traffic assignment literature often deal with similarity as well as uncertainty. ${ }^{2}$ The distinction between those two different meanings is hardly ever clear and we propose here to formalize properly the concept of a generalized fuzzy cost gathering both those semantics.

As already mentioned, a real cost is not directly perceived and handled by drivers who rather consider fuzzy costs based on the real value of the cost. Let us define $\widetilde{C}_{i}^{P}$ the fuzzy perception of a driver when cost on route $i$ is $C_{i}$. Its membership function is based on a similarity measure like in eq. 1:

$$
\forall x \in \Re, \mu_{\widetilde{C}_{i}^{p}}(x)=S\left(x, C_{i}\right)
$$

In order to introduce uncertainty, let us consider that traffic can be in several states $\omega$ and let us denote $\Omega$ the set of all those possible states. A state $\omega$ is actually a vector of traffic conditions giving the demand for travel on all OD pair, the capacity of the routes, the incidents, etc.

We are only interested in situations which we call "recurrent" and where the set $\Omega$ is not too big and corresponds to "normal" traffic conditions, occurring often, still unpredictable. In such situations we can suppose that:

- Drivers do not know what is the current state $\omega^{*}$, but they know the set $\Omega$ of all the possible states and the possibility $\pi(\omega)$ of each state $\omega$.

- They also know the real $\operatorname{cost} C_{i}(\omega)$ that would occur if any situation $\omega$ happened.

\footnotetext{
${ }^{2}$ Note that the preference concept is somewhat different and is rarely explicitly considered
} 
Such hypothesis are extending Wardrop's ones to situations with uncertainty.

In such a framework, a fuzzy generalized cost can be devised gathering both imprecision due to non-willingness of drivers to handle costs to the nearest second and uncertainty due to their incapability of predicting the state of traffic. Such a cost is the union of the possible perceived costs $\widetilde{C}_{i}^{P}(\omega)$ in each state $\omega$. Its membership function is given by:

$$
\begin{aligned}
\forall x \in \Re, \mu_{\widetilde{C}_{i}}(x) & =\max _{\omega \in \Omega} \min \left\{\mu_{\widetilde{C}_{i}^{p}(\omega)}(x) ; \pi(\omega)\right\} \\
& =\max _{\omega \in \Omega} \min \left\{S\left(x, C_{i}^{P}(\omega)\right) ; \pi(\omega)\right\}
\end{aligned}
$$

The membership level of an element $x$ to this fuzzy generalized cost thus represents something like the possibility level that the value $x$ is perceived by a driver.

It is interesting to note that even if the two natures of fuzziness have been considered separately, they cannot be extracted anymore from the final cost. Indeed, given a value $\mu$ of the membership function, it is impossible to come back to the initial cost $C_{i}$, neither to the possibility $\pi(\omega)$ of a situation or to the perceived cost $\widetilde{C}_{i}^{P}$.

\section{HANDLING FUZZY COSTS}

Once they are defined, with a precise and meaningful semantic, fuzzy costs have to be handled in order to choose the best route. We will show in this section that the nearly "classical" way of comparing fuzzy costs is only valid in regard to imprecision and not to uncertainty.

\subsection{Comparing fuzzy numbers}

In the traffic assignment literature, fuzzy costs are usually compared by using indices based on the possibility theory and calculating the possibility or the necessity that the (fuzzy) cost on a route is smaller than the ones of the other routes.

Such indices seem quite natural and easy to handle and very few authors refer to a theoretical background. Nevertheless, such a background exists and has to be considered. Dubois and Prade [5] have developed a comprehensive theory on comparing fuzzy numbers in the setting of possibility theory and it is important to remind several results such as the existence of 4 different indices for comparing two costs $M$ and $N$ :

$$
\operatorname{Poss}(M \leq N) \quad \operatorname{Nec}(M \leq N) \quad \operatorname{Poss}(M<N) \quad \operatorname{Nec}(M<N)
$$

All those indices may give different results and thus they have to be considered all together. It is also important to remember that they are to be handled cautiously because the inequality signs do not behave in the "classical" sense. For example, $M \leq N$ is not always equivalent to $N \geq M$; thus the complementary of $M \leq N$ is $M>N$ and not $N \leq M$ or $N<M$ as can be seen for example in [15]. This leads for example to:

$$
\operatorname{Poss}(M \leq N)=\operatorname{Poss}(\overline{M>N})=1-\operatorname{Nec}(M>N) \neq 1-\operatorname{Nec}(N \leq M)
$$




\subsection{Managing imprecision}

We have formally studied those indices for the traffic assignment problem in [9]. The basis of the model is to link the probability $p_{i}$ of choosing route $i$ with $I\left(\widetilde{C}_{i}\right)$, the value of the comparison index for the perceived cost on this route. The model we proposed was the simplest one. It assumes the proportionally between $p_{i}$ and $I\left(\widetilde{C}_{i}\right)$ and verifies the normality of probability $\left(\sum_{i} p_{i}=1\right)$ :

$$
p_{i}=\frac{I\left(\widetilde{C}_{i}\right)}{\sum_{j} I\left(\widetilde{C}_{j}\right)}
$$

The proportion $\gamma_{i}$ of traffic on route $i$ is then equal to this probability, due to the law of large numbers.

Such a model (or similar ones) has been used by several authors (even if they not always explicitly use the comparison indices or if they use only one of them) $[1,2,3$, $13,15]$.

The transformation we used from a possibility-based value into a probability measure may be criticized because of its lack of justification. Perinchery et al. [15] proposed a more valid transformation (when using the first index: Poss $\left(C_{i} \leq C_{j}\right)$ ) which is based on the principle of uncertainty invariance. Other methods could as well be considered based on other possibility-probability transformations (see [4] for a review on the links between those two theories).

Anyway, we think the above presented model (eq. 6) remains interesting because of its simple formulation. For example, its simplicity makes it possible to prove it is equivalent to the logit model when using specific membership functions [9]. This means that the logit model is a special case of this model when the similarity measure has a given shape.

\subsection{Managing uncertainty}

It is tempting to try to extend the previous model to the generalized fuzzy costs we introduced earlier, gathering both imprecision and uncertainty. The issue is then to verify that such a model corresponds to some valid individual behaviour in regard to choice making under uncertainty.

Actually, this decision making issue has not been explored because, as we already mentioned, generalized fuzzy costs are too compact to extract the uncertainty or imprecision parts from them. Nevertheless, we have discovered some incorrect behaviour of the model and this can prove the inconsistency of the underlying individual route choice model.

To illustrate the problem, let us consider a 3-routes network with one origin-destination. The routes are independent and their $\operatorname{costs} C_{i}$ do not depend on traffic but can take any value in $\left[C_{i}^{-} ; C_{i}^{+}\right]$, because of some random effect. Each triplet of value value corresponds to a (fully) possible situation $\omega$ (with $\pi(\omega)=1$ ). Drivers who are choosing between those routes are then facing uncertainty (because they do not know today's situation) and they handle fuzzy costs $\widetilde{C}_{i}$ such that:

$$
\forall x \in\left[C_{i}^{-} ; C_{i}^{+}\right], \quad \mu \widetilde{C}_{i}(x)=1
$$



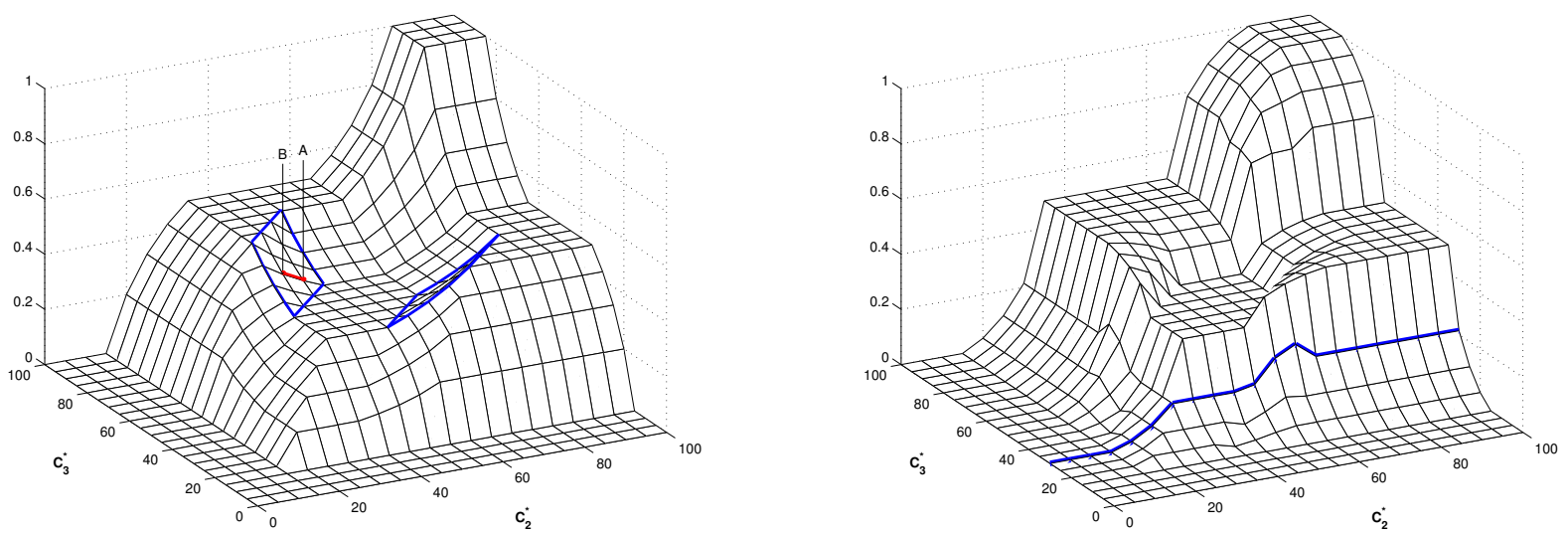

Figure 1: Variation of $p_{1}$, the probability that route 1 is chosen in regard to the cost on other routes (left: eq. 6; right: model of [15] with information conservation). An incorrect behaviour is observed in the enclosed zones: for example, $p_{1}(A)$ should be greater than $p_{1}(B)$

The probability $p_{i}$ to choose one route $i$ should increase when the $\operatorname{cost}\left(C_{j}^{-}\right.$or $\left.C_{j}^{+}\right)$on the other routes increases. Such a property seems quite natural but it is not always verified by the proposed model (eq. 6), as can be seen on figure 1 (on the left).

This problem does not seem to be linked with the possibility-probability transformation. Indeed, the transformation proposed by Perrincherry et al. has the same drawback (see fig. 1 on the right).

Actually it seems that the problem is rather due to the impossibility to order the routes when the fuzzy costs are not sharp (i.e. when there exist several $x$ such that $\mu \widetilde{C}_{i}(x)=$ 1). More details an be found in [10].

This implies that the same counter-intuitive behaviour could as well be observed without uncertainty but with non-discriminating similarity measures. So, in order to avoid such problem, similarity measures will be compelled to verify:

$$
\forall x, y \in \Re, \quad S(x, y)=1 \Longrightarrow x=y
$$

Finally, it seems that the basis of the route choice model are incorrect in situations with uncertainty and are to be modified. Actually, it is our opinion that the whole approach has to be revised and that imprecision and uncertainty cannot be considered together in a unique fuzzy cost gathering both aspects.

\section{TOWARDS A NEW TRAFFIC ASSIGNMENT MODEL}

We propose in this section some basis for the development of a new model, based on decision theory and handling both with imprecision and uncertainty, but with a valid individual route choice behaviour.

\subsection{Individual choice model}

For a given situation $\omega$, the cost on route $i$ is $C_{i}(\omega)$ and is supposed to be perceived by a driver as a fuzzy cost $\widetilde{C}_{i}^{P}(\omega)$ as defined by eq. 4 . 
In section 2.2 we introduced a preference measure $\phi$ between real values. It can be extended to fuzzy numbers and we can then define a preference measure on fuzzy perceived costs:

$$
\varphi\left(\widetilde{C}_{i}^{P}\right)=\max _{x \in \mathfrak{R}} \min \left\{\phi(x) ; \mu_{\widetilde{C}_{i}^{p}}(x)\right\}
$$

Without uncertainty, drivers are supposed to try to minimize their travel cost so that they will choose route $i^{*}$ with the highest preference: $\phi\left(C_{i^{*}}\right)=1$. If their perception is fuzzy, their behaviour remains identical and they will choose route $i^{*}$ such that $\varphi\left(\widetilde{C}_{i^{*}}^{P}\right)=1$.

The problem is to consider "recurrent situations" where drivers do not know the conditions they will encounter during their trip. So they have to consider the set of all the possible costs $\widetilde{C}_{i}^{P}(\omega)$. If one route is always preferred to the others, that is if $\forall \omega: \varphi\left(\widetilde{C}_{i}^{P}(\omega)\right)=1$, it means that this route is always the best one (whatever are the traveling conditions) and it can be chosen by a driver. But, generally, it is not the case and a decision model has to be defined, combining both preference and possibility levels. We use the following preference index for each route, considering all the possible conditions [8]:

$$
v_{i}=\inf _{\omega \in \Omega} \max \left\{1-\pi(\omega) ; \phi\left(\widetilde{C}_{i}(\omega)\right)\right\}
$$

This is a pessimistic index in the sense that it considers the worst (the less preferred) possible situations.

Every driver facing a set of routes with preference indices $v_{i}$ is then supposed to choose route $i^{*}$ such that $v_{i^{*}}=\max _{i} v_{i}$.

Such a decision model has been justified by $[6,8]$ in the frame of decision theory under uncertainty by a set of axioms similar to those given by Savage [16] or by von Neuman and Morgenstern [17] to justify the expected utility criterion.

\subsection{Traffic assignment model}

The proportion $\gamma_{i}$ of drivers choosing route $i$ is calculated by aggregating the individual choices based on the preceding individual model. So, if we consider a homogeneous population with identical drivers, we can write:

$$
v_{i}<\max _{j} v_{j} \Longrightarrow \gamma_{i}=0
$$

We also have to consider the fact that costs depend on the traffic on the routes which is a consequence of individual choices based on the same costs. However, in "recurrent" situations, we can suppose that an equilibrium is observed where the ex post realized costs are coherent with the ex ante predicted possible costs.

In other words, a vector $\gamma$ of traffic proportions may lead, through a traffic flow model, to a set of real costs vectors $\{C(\omega)\}_{\omega \in \Omega}$ (depending on the set $\Omega$ of possible conditions). Those real costs are perceived by drivers as fuzzy costs (eq. 4) and leads to a vector $v$ of preferences for the routes (eq. 8). The equilibrium condition means that those preferences verifies eq. 9 with the initial traffic proportion vector $\gamma$. 


\section{CONCLUSION AND PERSPECTIVES}

The model that has just been presented gives a comprehensive framework for the treatment of imprecision, preference and uncertainty. Imprecision is handled by the way of fuzzy costs, preference by a preference measure and uncertainty by a possibility distribution of the possible traffic conditions. They are all incorporated in an individual route choice model which is justified by a sound axiomatic.

Of course, the study of such a model still remains to be done since this paper was only an introduction. In particular, the influence of each element (imprecision, preference and uncertainty) is to be explored as well as the global traffic assignment model. We intend in a future paper to try to define some extension to the Wardrop principle in "recurrent" situations where everything is not known by drivers.

The individual behaviour has also to be validated. Even if it has been theoretically justified, it is important to verify that it corresponds (or not) to the real behaviour of drivers.

But above all, this paper was willing to demonstrate that fuzzy models could be built with solid basis and a precise semantic. Of course this implies also some rigor and discipline, but it is the necessary price to benefit from the advantages of a new theory.

\section{REFERENCES}

[1] Takamasa Akiyama and Testuya Kawahara. Traffic assignment model with fuzzy travel time information. In 9th mini EURO Conf:: Fuzzy sets in Traffic and Transport Systems, Budva, Yougoslavia, September 1997.

[2] Michel Bierlaire, Didier Burton, and Tsippy Lotan. On the behavioural aspects of modal choices. GRT Report 93/22, Facultés Universitaires ND de la Paix, Namur, Belgique, 1993.

[3] Huey-Kuo Chen and Mei-Shiang Chang. A fuzzy dynamic optimal route choice model. In Transportation Research Board annual meeting, Washington, January 1998.

[4] Didier Dubois, Hung T. Nguyen, and Henri Prade. Possibility theory, probability and fuzzy sets: misunderstanding, bridges and gaps. In Didier Dubois and Henri Prade, editors, Fundamental of fuzzy sets, The handbook of fuzzy sets, pages 343438. Kluwer academic publisher, 1999.

[5] Didier Dubois and Henri Prade. Ranking fuzzy numbers in the setting of possibility theory. Information sciences, 30(2):183-224, 1983.

[6] Didier Dubois and Henri Prade. Possibility theory as a basis for qualitative decision theory. In 14th Int. joint conference on artificial intelligence, pages 1924-1930, Montreal, Canada, 1995.

[7] Didier Dubois and Henri Prade. The three semantics of fuzzy sets. Fuzzy Sets and Systems, 90:141-150, 1997.

[8] Didier Dubois, Henri Prade, and Régis Sabbadin. Decision-theoretic foundations of qualitative possibility theory. European Journal of Operational Research, 128:459478 , 2001. Invited review. 
[9] Vincent Henn. Fuzzy route choice model for traffic assignment. In 9th mini EURO Conf.: Fuzzy sets in Traffic and Transport Systems, Budva, Yougoslavia, September 1997. (Also in Fuzzy Sets and Systems, 116(1):77-101, 2000.)

[10] Vincent Henn. Traffic information and traffic assignment: towards a fuzzy model. PhD thesis, Saint-Étienne University, France, June 2001. In french.

[11] Tsippy Lotan. Modelling route choice behaviour in the presence of information using concepts of fuzzy sets theory and approximate reasoning. PhD thesis, Massachussets Institute of Technology, 1992.

[12] Tsippy Lotan and Hari N. Koutsopoulos. Approximate reasonning models for route choice behavior in the presence of information. In Carlos F. Daganzo, editor, Transportation and Traffic Theory (Proc. of the 12th International Symposium on the Theory of Traffic Flow and Transportation), pages 71-88. Elsevier, 1993.

[13] Shinkoh Okada and Timothy Soper. A shortest path problem on a network with fuzzy arc lengths. Fuzzy Sets and Systems, 109:129-140, 2000.

[14] S. A. Orlovsky. Decision-making with a fuzzy preference relation. Fuzzy Sets and Systems, 1:155-167, 1978.

[15] Vijay Perincherry, Shinya Kikuchi, and Mauro Dell'Orco. Use of possibility theory when dealing with uncertaity in modelling choice. In Euro Working Group on Transportation meeting, Rome, 2000.

[16] L. J. Savage. The foundations of statistics. Wiley, New York, 1954.

[17] J. von Neumann and O. Morgenstern. Theory of games and economic behavior. Princeton Univ. Press, Princeton, NJ, 1944.

[18] Lotfi A. Zadeh. Fuzzy sets. Information and control, 8:338-353, 1965.

[19] Lotfi A. Zadeh. Similarity relations and fuzzy ordering. Information sciences, 3:177-200, 1971. 